\title{
mantumancm \\ Phenytoin: an old but effective antiarrhythmic agent for the suppression of ventricular tachycardia
}

Louis W Wang BSc(Med), MB BS, MM Cardiology Advanced Trainee ${ }^{1}$

\section{Rajesh N Subbiah} MB BS, PhD, FRACP, Consultant Cardiologist and Co-Director of Cardiac Electrophysiology, and Associate Professor of Medicine ${ }^{2}$

Michael J Kilborn BM BCh, DPhil, FRACP, Consultant Cardiologist
Con BCh, DPhil, FRACP, and Director of Arrhythmia Service, ${ }^{3}$ and Clinical Associate Professor in Medicine $^{4}$

Richard F Dunn MB BS, FRACP, FCSANZ Consultant Cardiologist

1St Vincent's Hospital Sydney, NSW.

2 St Vincent's Clinical School, University of New South Wales, Sydney, NSW.

3 Royal Prince Alfred Hospital, Sydney, NSW.

4 Central Clinical School University of Sydney, Sydney, NSW.

5 Bankstown-Lidcombe Hospital, Sydney, NSW.

louis.wang@ unsw.edu.au

doi: 10.5694/mjal3.10224

\section{Clinical record}

A 73-year-old man presented with recurrent ventricular tachycardia (VT) on a background of severe, non-ischaemic dilated cardiomyopathy (ejection fraction, 20\%) and cardiac resynchronisation therapy with an implantable cardioverter defibrillator (ICD). Over the previous 6 months, he had frequent appropriate ICD shocks for VT after unsuccessful antitachycardia pacing. A coronary angiogram was normal. Amiodarone had been successful in reducing VT burden and ICD shocks, but was ceased because of amiodarone-induced thyrotoxicosis. Shortly after discontinuing amiodarone, there was a significant increase in VT burden and appropriate ICD shocks. An endocardial mapping and VT ablation procedure had been performed, but this was unsuccessful and suggested an epicardial source of VT. The patient also underwent subsequent epicardial mapping and VT ablation using a CARTO 3 (Biosense Webster) electroanatomical mapping system, which localised the source of VT to a diffuse area of the basal anterolateral segment of the left ventricle. A total of 17 ablations were performed. However, VT was still inducible after these ablations, and the procedure was terminated after 6.75 hours. Repeat epicardial VT ablation was performed 3 months later, although this was also unsuccessful. Since the frequency of VT had increased after resynchronisation therapy, the pacing mode was converted to dual-chamber atrioventricular pacing with right ventricular pacing only (right ventricular pacing, 85\%; atrial pacing, 49\%), in case left ventricular pacing was proarrhythmic. To allow reintroduction of amiodarone for $\mathrm{VT}$ suppression, the patient then underwent total thyroidectomy and, after an uneventful postoperative recovery, he was discharged, having been restarted on regular oral amiodarone. His medications included amiodarone $200 \mathrm{mg}$ three times daily, carvedilol $50 \mathrm{mg}$ twice daily, spironolactone $25 \mathrm{mg}$ daily, frusemide $40 \mathrm{mg}$ daily, irbesartan $150 \mathrm{mg}$ daily, atorvastatin $40 \mathrm{mg}$ daily, magnesium supplementation, and warfarin.

The next day, he returned to his local district hospital after experiencing another ICD shock. He was found to be in his usual paced rhythm (Box 1, A), with frequent runs of non-sustained monomorphic VT (Box 1, B). Given that he was already on amiodarone, a lignocaine infusion (maintenance rate, $2 \mathrm{mg} / \mathrm{min}$ ) was added. This resulted in suppression of $\mathrm{VT}$, but was complicated by confusion and perioral paraesthesia, which persisted despite reduction of infusion rate. Lignocaine was stopped after 24 hours, but this was followed soon afterwards by a VT storm.

Due to the previous success of lignocaine (a class IB antiarrhythmic drug) in controlling VT, trial of another agent in this class was warranted. Mexiletine or tocainide were not readily available; however, we noted that phenytoin had class IB activity and was readily available on the ward. Phenytoin was initially administered as a loading dose $(15 \mathrm{mg} / \mathrm{kg}, 1 \mathrm{~g} / \mathrm{h})$ and was immediately successful in suppressing the VT storm. However, at the end of the infusion, the patient developed nystagmus, drowsiness and hypotension. QRS duration lengthened (Box 1, C). Bradycardia (heart rate, 35-45 beats/min) resulting from intermittent loss of capture of right ventricular pacing was also noted on continuous telemetry (Box $2, A)$. This was confirmed on pacemaker-ICD interrogation. The right ventricular pacing threshold had increased from $1.4 \mathrm{~V}$ at $0.4 \mathrm{~ms}$ before phenytoin to $3.0 \mathrm{~V}$ at $0.4 \mathrm{~ms}$ after phenytoin (Box 3 ). Previous right ventricular pacing output had been set at $2.8 \mathrm{~V}$ at $0.4 \mathrm{~ms}$, below the new threshold, which explained the frequent loss of capture. The pacing amplitude was increased to accommodate the effects of phenytoin loading and ongoing maintenance therapy (Box 2, B). The defibrillation threshold (21 $\mathrm{J}$ at time of implantation) was not initially rechecked, but due to the concern that this may also have been increased by phenytoin, the shock setting was increased to the maximum (41 J). Regular oral phenytoin (100 mg three times daily) was commenced, with trough levels confirming that the patient was in the therapeutic range (Day 7 after commencement, $52 \mu \mathrm{mol} / \mathrm{L}$; Day 14 after commencement, $70 \mu \mathrm{mol} / \mathrm{L}$; reference interval, $40-100 \mu \mathrm{mol} / \mathrm{L}$ ).

Repeat pacemaker-ICD interrogation performed 9 days after phenytoin commencement demonstrated sustained suppression of ventricular ectopy, with only one further episode of VT, which was successfully terminated by antitachycardia pacing. The pacing thresholds stabilised at a higher level 12 days after phenytoin commencement (Box 3 ) and remained stable thereafter. A defibrillation threshold test performed 46 days after phenytoin commencement showed no change to the threshold. henytoin, a widely used anticonvulsant, has class IB antiarrhythmic drug (AAD) properties, and is a potential option for patients with refractory ventricular arrhythmia when other agents are contraindicated or unavailable. In patients with frequent ventricular arrhythmia, AADs are often used to reduce the frequency of arrhythmia and implantable cardioverter defibrillator (ICD) shocks. Amiodarone is often used as first-line therapy. Although class I AADs are generally not pre- scribed for patients with ischaemic heart disease or cardiomyopathy, they are occasionally used in combination with amiodarone in patients who are refractory to amiodarone monotherapy, especially those with ICDs, as this provides some protection against potential proarrhythmic side effects. ${ }^{1}$

Phenytoin has class IB antiarrhythmic properties due to its effects on sodium channels in cardiac myocyte and Purkinje fibre cell membranes. ${ }^{2}$ It reduces the maximum 


\section{Electrocardiograms}

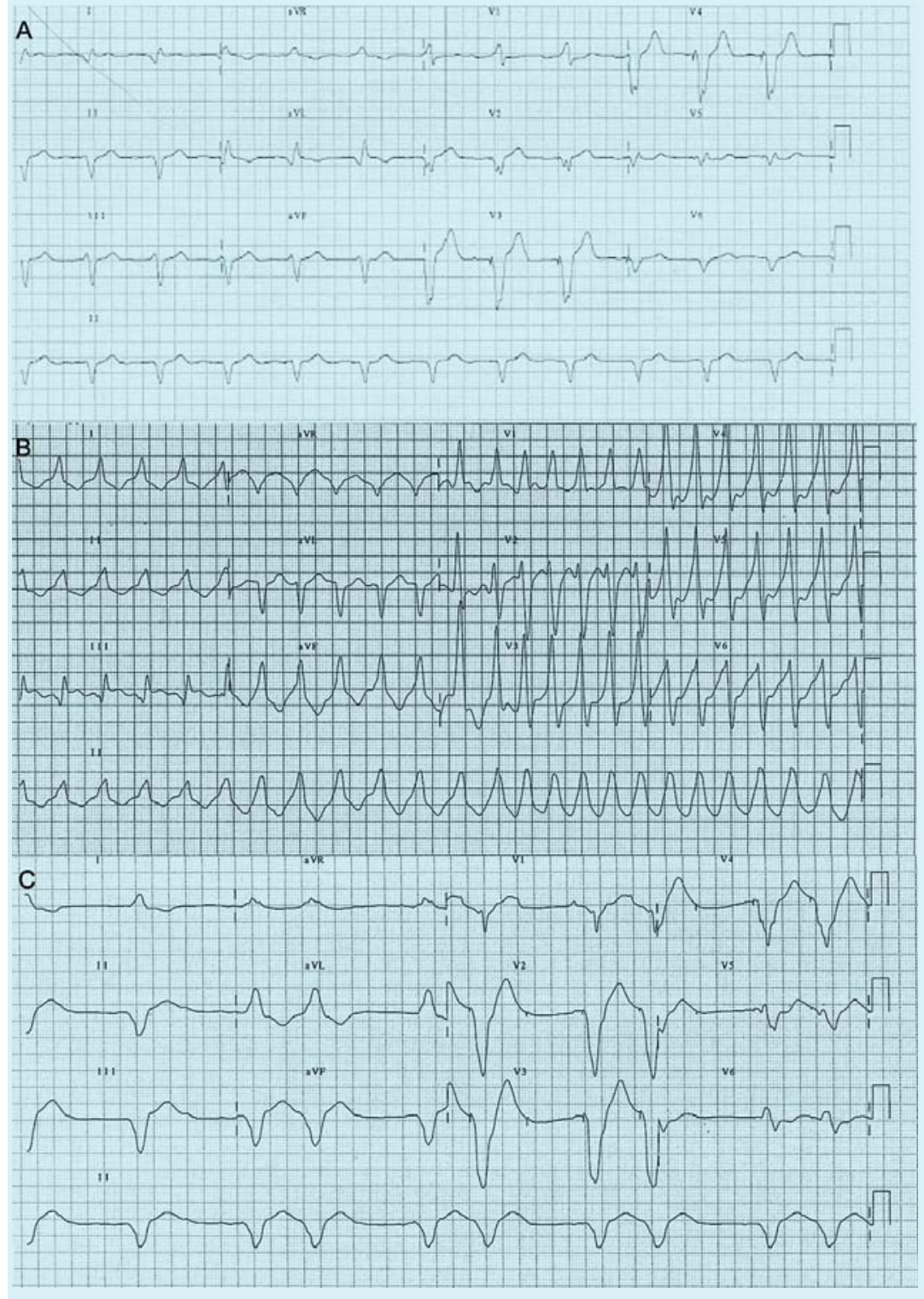

Twelve-lead electrocardiograms showing left bundle branch block morphology of the patient's right ventricular paced rhythm (70 beats/min) (A); ventricular tachycardia originating from the left ventricle (B); and, following phenytoin loading, suppression of $V T$ and return to right ventricular paced rhythm, but with widening of the QRS complex, secondary to the effect of phenytoin (C).

\section{Telemetry}

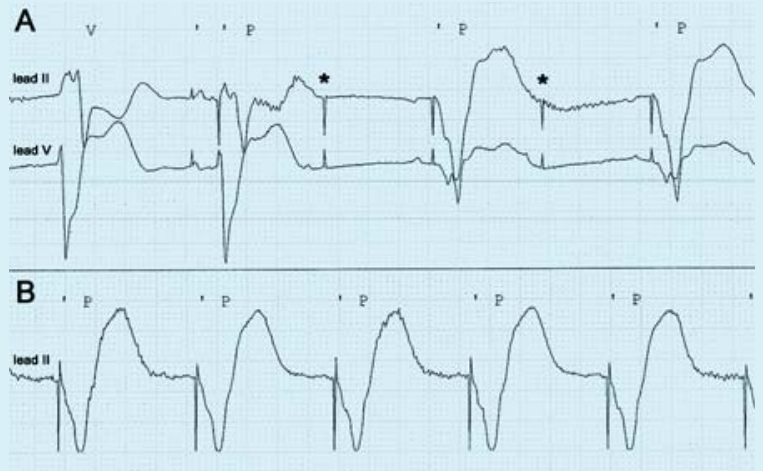

Telemetry showing intermittent loss of capture of right ventricular pacing $(*)(A)$; and restoration of normal ventricular capture after an increase in right ventricular pacing output (B).

rate of depolarisation of the cardiac action potential and increases the effective refractory period. Therefore, it is particularly effective in inhibiting ventricular ectopy, especially in an ischaemic or damaged myocardium. ${ }^{3}$ Although used as an AAD from the 1950s to the 1970 s $^{4-6}$ phenytoin has become obsolete in recent decades due to the arrival of newer and less toxic agents. Recent case reports have documented its successful use in controlling idiopathic ventricular fibrillation in a young $\operatorname{man}^{7}$ and refractory idiopathic ventricular tachycardia (VT) in a newborn, ${ }^{8}$ but these reports have not documented the potential dangers associated with its use.

Although phenytoin was successful in suppressing VT in our patient, this case report highlights several important learning points. Class I AADs, including phenytoin, can increase pacing threshold through use-dependent inhibition of sodium channel function. ${ }^{9}$ Most pacemakers have algorithms that allow for potential variations in pacing thresholds. ${ }^{10}$ However, AAD-induced elevation of pacing threshold may become problematic in circumstances where the pacing threshold is already elevated, such as following ICD shock or, as in our patient, the recent use of other class I AADs. AADs can also increase the defibrillation threshold, increase VT cycle length (potentially resulting in VT undersensing) and reduce antitachycardia pacing efficacy. ${ }^{2}$ The effect of AADs on pacing threshold can be temporarily ameliorated by increasing the settings

3 Effect of phenytoin on pacing threshold

\begin{tabular}{|c|c|c|c|c|}
\hline Lead & Before loading* & Immediately after loading ${ }^{\dagger}$ & After 9 days ${ }^{\ddagger}$ & After 12 days $^{\S}$ \\
\hline \multicolumn{5}{|l|}{ Right ventricular } \\
\hline Pacing impedance & $1043 \Omega$ & $1067 \Omega$ & $1074 \Omega$ & $1098 \Omega$ \\
\hline Pacing threshold & $1.4 \mathrm{~V}$ at $0.4 \mathrm{~ms}$ & $3.0 \mathrm{~V}$ at $0.4 \mathrm{~ms}$ & $\begin{array}{l}4.0 \mathrm{~V} \text { at } 0.4 \mathrm{~ms} \text { or } \\
2.8 \mathrm{~V} \text { at } 1.0 \mathrm{~ms}\end{array}$ & $\begin{array}{l}3.3 \mathrm{~V} \text { at } 0.4 \mathrm{~ms} \text { or } \\
2.2 \mathrm{~V} \text { at } 1.0 \mathrm{~ms}\end{array}$ \\
\hline \multicolumn{5}{|l|}{ Atrial } \\
\hline Pacing impedance & $695 \Omega$ & $710 \Omega$ & $671 \Omega$ & $657 \Omega$ \\
\hline Pacing threshold & $0.8 \mathrm{~V}$ at $0.4 \mathrm{~ms}$ & $1.2 \mathrm{~V}$ at $0.4 \mathrm{~ms}$ & $1.1 \mathrm{~V}$ at $0.4 \mathrm{~ms}$ & $1.1 \mathrm{~V}$ at $0.4 \mathrm{~ms}$ \\
\hline
\end{tabular}

* Right ventricular pacing output set at $2.8 \mathrm{~V}$ at $0.4 \mathrm{~ms}$ and atrial pacing output at $1.3 \mathrm{~V}$ at $0.4 \mathrm{~ms}$. $\dagger$ After loss of capture and pacemaker interrogation, right ventricular pacing output was increased to $7.5 \mathrm{~V}$ at $1.0 \mathrm{~ms}$ and atrial pacing output to $2.5 \mathrm{~V}$ at $0.4 \mathrm{~ms}$ to guarantee capture. $¥ A$ fter an increase in right ventricular threshold values, pacing output was temporarily increased to $7.5 \mathrm{~V}$ at $2.0 \mathrm{~ms}$ for 3 days, with repeat follow-up after 3 days. $\$$ After stabilisation of pacemaker threshold values, right ventricular pacing output was reduced to $5.0 \mathrm{~V}$ at $1.0 \mathrm{~ms}$, with an estimated battery life of 6.5 years. 


\section{Lessons from practice}

- Phenytoin has class IB antiarrhythmic drug properties and is a potential treatment option for patients with refractory ventricular arrhythmia when other agents have failed or are unavailable.

- However, phenytoin has a narrow therapeutic range and the potential for multiple drug interactions.

- Phenytoin administration may also result in elevation of pacemaker threshold. Care should be taken with loading doses in patients with cardiomyopathy or who are pacemaker-dependent, because of the potential for loss of pacemaker capture.

to levels that guarantee capture but may require readjustment to conserve battery life in the long term. Phenytoin exhibits zero-order pharmacokinetics, is susceptible to multiple drug interactions and has a narrow therapeutic window. Vertigo, ataxia, headache and nystagmus are common early side effects. At higher plasma concentrations, marked confusion and sedation may result. All of these changes occur acutely and are rapidly reversible. Cardiovascular side effects include bradycardia, hypotension and, occasionally, exacerbation of VT. Our patient developed side effects despite a conventional loading protocol used in treating status epilepticus. Factors that may have contributed to toxicity were the presence of severe cardiomyopathy — which may have altered tissue distribution properties and resulted in a higher availability of unbound drug - and recent lignocaine administration, although this had been ceased 6 hours earlier. Rapid loading of phenytoin for VT has been associated with fatal arrhythmogenic complications ${ }^{3}$ and should therefore be cautiously performed.

Phenytoin, a commonly used drug in the treatment of status epilepticus, is an old but effective treatment option in patients with malignant VT or electrical storm. Although it is readily available and is successful in terminating and suppressing malignant VT, appropriate caution must be taken with its use, especially with regard to the increased likelihood of drug toxicity in patients with cardiomyopathy and its important effects on pacemaker and ICD function. These reported effects of phenytoin on pacemaker function are not only of interest to cardiologists caring for patients with ventricular arrhythmia, but also to neurologists, and emergency and general physicians who use phenytoin to terminate seizures. Owing to the effects of phenytoin on pacemaker threshold and the attendant risks of loss of pacemaker capture, care should be taken with loading doses of phenytoin in patients who are pacemakerdependent.

\section{Competing interests: No relevant disclosures.}

1 Van Herendael H, Pinter A, Ahmad K, et al. Role of antiarrhythmic drugs in patients with implantable cardioverter defibrillators. Europace 2010; 12: 618-625.

2 Atkinson AJ Jr, Davison R. Diphenylhydantoin as an antiarrhythmic drug. Annu Rev Med 1974; 25: 99-113.

3 Kowey PR. Pharmacological effects of antiarrhythmic drugs. Review and update. Arch Intern Med 1998; 158: 325-332.

4 Leonard WA. The use of diphenylhydantoin (dilantin) sodium in the treatment of ventricular tachycardia. AMA Arch Intern Med 1958; 101: 714-717.

5 Conn RD. Diphenylhydantoin sodium in cardiac arrhythmias. N Engl J Med 1965; $272: 277-282$.

6 Eddy JD, Singh SP. Treatment of cardiac arrhythmias with phenytoin. Br Med J 1969; 4: 270-273.

7 Golian M, Bhagirath KM, Sapp JL, et al. Idiopathic ventricular fibrillation controlled successfully with phenytoin. J Cardiovasc Electrophysiol 2011; 22: 472-474.

8 Sun J, Shah N, Karpawich PP, Humes R. Refractory idiopathic ventricular tachycardia in a newborn treated successfully with phenytoin: old therapies are still effective in the current era. Pediatr Cardiol 2011; 32: 76-77.

9 Brode SE, Schwartzman D, Callans DJ, et al. ICD-antiarrhythmic drug and ICD-pacemaker interactions. J Cardiovasc Electrophysiol 1997; 8: 830-842.

10 Beadle R, Williams L, Lim HS. Drug-implantable cardioverter-defibrillator interactions. Expert Rev Cardiovasc Ther 2010; 8:1267-1273. 